\title{
TABLE OF ARTICLES OF THE CONVENTION, THE 1994 AGREEMENT RELATING TO THE IMPLEMENTATION OF THE CONVENTION, THE STATUTE AND THE RULES OF THE TRIBUNAL
}

1982 United Nations Convention on the Law of the Sea

Art 1(1).1 1.015

Art 1(2).1 3.027

Art 1(2).2 3.027

Art 2 3.046

Art 15 $1.049,3.061,3.166,5.035$

Art 18(1) 3.047

Art 32 3.047

Art 33 3.183

Part V 1.017

Art 55 3.046

Art 56 $3.046,3.183,5.054,5.063$

Art 56(1) 5.063

Art 58 $1.046,3.053,3.183,5.054$

Art 58(3) 5.066

Arts 61-68 5.063

Art 62(4) $5.063,5.066$

Art 64 1.074, 3.041, 3.103, 3.174, $5.064,5.072$

Art 73 $3.045,3.130,3.177,3.186$, $4.036,5.054$

Art 73(2) ......... 3.132, 3.134, 3.176, 4.250 Art 73(3) .................... 3.057, 3.136, 3.170 Art 73(4) 3.136

Art 74 ... 1.049, 3.061, 3.098, 3.166, 5.004 Art 74(1) $1.023,3.099$ Art 74(3) 1.023

Art 76 $2.073,3.040,3.046,5.004$ $5.016,5.017$

Art 76(8) 5.016

Art 83 ... 1.049, 3.061, 3.098, 3.166, 5.004 Art 83(1) $1.023,3.099$ Art 83(3) 1.023
Art 87 $3.045,3.047,3.167,4.036$

Art 90 3.047

Art 91 3.192, 3.195, 3.196, 4.207, $5.046,5.048$

Art 91(1) 3.197, 5.045

Art 94 $5.049,5.050$ Art 106 3.198

Art 110 $3.186,3.198,5.054$

Art 111 $3.183,3.198$

Art 116 $3.167,4.269$

Arts 116-119 $1.074,3.041,3.103$, $3.174,5.064,5.072$

Art 117 3.167

Art 121 $3.040,3.063$

Art 123 5.064

Part XI 1.017, 1.018, 1.019, 1.033, 1.038, 2.075, 2.079, 3.002, 3.003, 3.030, 3.032, 3.116, 3.119, 3.121, 3.123, 3.126, 3.128, 3.172, 3.173, $3.207,3.240,3.241,4.003,4.219$, 4.266, 4.267, 4.273, 5.074, 5.076, $5.078,6.003$

Art 136 $1.018,3.172$

Art $137(2)$ $3.173,5.080$ Art 139 $5.067,5.075$

Art 139(1) $5.028,5.075$

Art 139(2) ...... 5.075, 5.077-5.079, 5.081, 5.082

Art 153(2) ...... 3.124, 3.126, 3.127, 4.047, $5.076-5.077$

Art 153(4) $5.069,5.077$ Art 159(10) $3.205,4.308$

Art 162 $3.207,4.266$ Art 168(2) 3.127 
Art 185 $3.128,4.266$

Art 185(2) 4.266

Arts 186-191 2.075

Art 187 $3.009,3.017,3.032$, 3.089, $3.117,3.120-3.128,3.173,4.033$, $4.273,4.275$

Art 187(a) $2.079,3.121,3.122$

Art 187(b) 3.123

Art 187(c) ....... 3.125, 4.267, 4.273, 4.274

Art 187(d) 3.126

Art 187(e) 3.127

Art 187(f) 3.128

Art 188 $3.009,3.089,3.117,4.033$

Art 188(1) $2.079,3.122$

Art 188(2) $3.125,4.267,4.268$

Art 189 3.118

Art 190 $4.273,4.276$

Art 190(1) $4.271,4.275$

Art 190(2) 4.274

Art 191 3.033, 3.173, 3.204,

$3.206-3.208,4.308,4.309,4.315$ Part XII .......... 1.017, 5.011, 5.024, 5.064, 5.065

Art 192 $3.167,5.064,5.065,5.071$

Art 194 $3.169,3.170,5.064$

Art 197 $1.071,5.064$

Art 200 5.064

Arts 207, 208 5.064

Art 218(1) 5.071

Art 220(6) 3.132

Art 220(7) $3.132,4.250$

Art 224 5.054

Art 224 5.055

Art 226 $3.045,3.132,4.036,4.250$

Art 230(2) 3.170

Art 235(1) 5.075

Art 237 2.085

Art 245 $3.045,4.036$

Art 246 $1.046,1.047,3.055$

Art 253 $1.046,1.047,3.055$

Part XV .......... 1.016, 1.022, 1.026-1.028, 1.031, 1.033, 1.035, 1.051, 1.055, 1.057, 1.059, 1.064, 1.066, 1.067, 1.071, 1.073, 1.076, 1.077, 2.071, 3.001, 3.007, 3.008, 3.018, 3.028, 3.036, 3.043, 3.044, 3.048, 3.050, 3.051, 3.057, 3.065, 3.067-3.072,
3.078, 3.079, 3.082-3.084, 3.090, 3.091, 3.094, 3.098, 3.099, 3.101, 3.106, 3.109-3.111, 3.113, 3.114, 3.119, 3.132, 3.161, 3.162, 3.165, 3.181, 3.234, 4.003, 4.027, 4.028, 4.031, 4.222, 4.335, 4.339, 5.003, $5.007,5.019,6.003,6.008,6.009$

Art 279 1.023

Art 280 $1.024,1.025,1.056$

Art 281 1.025-1.027, 1.067, 3.007, 3.071, 3.090, 3.101-3.109, 5.007, 5.008

Art 282 $1.028,3.007,3.071,3.090$, 3.110-3.115

Art 283 $1.031,3.007,3.018,3.019$ 3.042, 3.071, 3.090-3.100, 3.166, 4.022

Art 283(1) $1.029,3.091,3.098$ Art 283(2) ....... 1.030, 3.091, 3.099, 3.100 Art 284 $1.032,4.003$

Art 285 1.033

Art 286 $1.034,3.050,3.068,3.079$, $3.165,3.167,5.072$

Art 287 $1.035,1.036,1.038-1.044$, 1.061, 1.063, 2.111, 2.126, 3.001, 3.002, 3.008, 3.019, 3.035, 3.043, 3.060, 3.071, 3.072-3.081, 3.117, 3.141, 3.144, 3.159, 3.215, 4.015, 4.028, 4.033, 4.049, 4.181, 4.216, 6.009

Art 287(1) ..... 1.037, 1.066, 3.069, 3.074, $3.078,3.079,3.081$

Art 287(2) $1.038,3.117$

Art 287(3) $1.040,3.076,3.114$

Art 287(4) ....... 1.039, 3.073, 3.074, 3.078 Art 287(5) $1.040,3.076$ Art 287(6), (7) 1.041

Art 288 3.002, 3.006, 3.017, 3.035, $3.159,3.215$

Art 288(1) $3.007,3.040,3.054$

Art 288(2) $3.213,3.215,4.339$

Art 288(3) $3.116,3.210$

Art 288(4) 3.020

Art 289 2.071-2.074, 2.138, 4.149, 4.279

Art 290 $1.038,3.155,4.068,5.010$ 5.011, 5.070, 5.071 
Art 290(1) ...... 3.139, 3.142, 3.143, 3.147, $3.154,3.162,4.028,4.141,4.165$, $4.169,4.174,5.011$

Art 290(2) 4.289

Art 290(3) $4.166,4.172$

Art 290(5) ...... 1.038, 3.010, 3.047, 3.065, $3.089,3.128,3.139-3.155,3.162$, 3.189, 4.014, 4.019, 4.022, 4.033, 4.141, 4.165, 4.168, 4.170, 4.171, 4.174, 4.296, 5.011, 5.021

Art 290(6) $3.158,4.284,4.289$ Art 292 1.038, 2.094, 3.010, 3.024, 3.066, 3.075, 3.089, 3.129-3.138, 3.175-3.177, 3.179, 3.183, 3.187, 3.188, 3.194, 3.200, 4.014, 4.033, 4.068, 4.144, 4.236, 4.238, 4.240, $4.250,5.021,5.041$ Art 292(1) ....... 3.131, 3.200, 4.249, 5.041 Art 292(2) ................... 3.024, 3.137, 4.139 Art 292(3) ...... 3.024, 3.067, 3.136, 3.176, $3.177,4.111,4.248$ Art 292(4) $4.252,4.284,4.295$

Art 293 $3.003,3.235,3.238,3.239$

$3.242,5.023,5.039$

Art 293(1) $1.067,3.234$

Art 293(2) 3.234

Art 294 . 3.060, 4.007, 4.181, 4.185, 4.188 Art 294(1), (2) 3.060 Art 294(3) $3.060,4.188 .4 .189$

Art 295 $3.181,5.052$

Art 296 4.283

Art 296(2) 5.029

Art 297 $1.045,1.055,3.006,3.018$

$3.050,3.051,3.054,3.057-3.060$, 3.064, 3.068, 3.083, 3.084, 4.114, $4.181,4.183,4.184,4.186-4.188$ Art 297(1) $1.046,3.053,3.054$

Art 297(2) ...... 1.046, 1.049, 1.047, 3.054, $3.055,3.057,3.061,3.064,3.065$, 3.067

Art 297(3) ...... 1.046, 1.047, 1.049, 3.054, $3.056-3.058,3.061,3.064-3.067$

Art 298 1.043. 1.045, 1.049-1.051, $1.053-1.055,1.063,1.064$, 2.070-2.074, 2.111, 2.130, 3.006, 3.018, 3.048, 3.050, 3.051, 3.061,
$3.062,3.064,3.067,3.068,3.083$, $3.084,3.100,4.015,4.049,4.114$ Art 298(1) ...... 1.049, 1.051, 1.052, 1.054, 2.130, 3.048, 3.062, 3.061, 3.064-3.067

Art 298(6) 1.049

Art 299 $1.045,1.055,3.050,3.051$

Art 300 $4.036,5.040,5.054$

Art 303 $3.045,4.036$

Art 304 5.075

Art 305 3.027

Art 309 $1.043,3.052$

Art 311 3.173

Art 313 2.024

Art 318 3.215

Art 319 2.133

Annex III $3.121,3.126,3.127,5.075$

Art 4 $3.126,5.075,5.077$

Art 5 3.126

Art 13 3.126

Art 14 3.127

Art 22 $3.126,3.127,5.075,5.077$

Annex IV 3.121

Annex V $1.032,1.047,1.048,1.052$ $1.053,3.046$

Annex VI ....... 1.036, 2.002, 2.007, 2.024, $2.075,2.078,2.079,3.002,3.116$ $3.125,3.215,3.216,4.003-4.005$ see also Statute of the Tribunal

Annex VII ...... 1.036, 1.040, 1.044, 1.057, 1.076, 2.002, 2.039, 2.040, 2.054, 2.055, 2.057-2.060, 2.090, 2.092, $3.035,3.046,3.054,3.063,3.069$, 3.078, 3.082, 3.099, 3.104, 3.109, 3.114, 3.144, 3.155, 3.189, 4.092, 4.165, 4.211, 5.006, 5.013, 5.042, $5.072,6.009,6.018$

Art 1 2.055

Art 2 $2.055,2.057-2.059$

Art 3 $2.059,2.092,3.155$

Art 3(b) 2.055

Art 3(c) 2.056

Art 3(d) 2.057

Art 3(e) 2.058

Art 5 $3.035,4.211$

Annex VIII ..... 1.036, 1.057, 2.071, 2.138, $3.035,3.069$ 
Art 2 $2.071,2.138$

Annex IX $2.111,3.028,5.087,5.088$ Art 4 2.026

Art 6 5.088

Art 7 $1.037,2.111,3.028$

1994 Agreement relating to the

Implementation of Part XI of the United Nations Convention on the

Law of the Sea ..... 1.018, 1.019, 3.119, 3.121, 3.126, 3.207, 3.240, 3.241, 4.007, 5.075, 5.076

\section{Statute of the Tribunal (Annex VI to the} 1982 United Nations Convention on the Law of the Sea)

see also Annex VI to the Convention

Art 1(1) 2.002

Art 1(2) 2.006

Art 1(3) 2.016

Art 2 $2.027,2.062,2.063,3.017$

Art 2(2) 2.022

Art 3(1) 2.027

Art 3(2) 2.027

Art 4(3) 2.024

Art 4(4) 2.025

Art 5 2.036

Art 5(1) 2.032

Art 5(2) 2.032

Art 6 2.038

Art 7 2.039

Art 8 $2.040,2.062$

Art 9 2.040

Art 10 2.063

Art 11 $2.035,2.061,2.062$

Art 12(1) 2.003

Art $12(2)$ 2.004

Art 12(3) 2.044

Art 14 $2.066,2.076$

Art 15 2.066, 2.079, 2.080, 3.009, $3.122,4.002,4.256-4.258,4.261$ Art 15(1) ........ 2.066, 2.080, 2.081, 2.092, $3.009,4.256,4.258,4.259$ Art 15(2) ........ 2.066, 2.080, 2.089, 2.092, $3.009,4.256-4.258$ Art 15(3) ........ 2.066, 2.080, 2.093, 3.009, $4.256,4.258,4.259$

Art 15(4) 2.077 Art 15(5) $2.080,4.283$
Art 16 4.004

Art 17 ... 2.061, 2.063, 2.079, 4.057, 4.319 Art 17(4) 2.066

Art 17(5) 2.065

Art 17(6) 2.062

Art 18 $2.132,2.046$

Art 18(1), (5) 2.042

Art 18(7) 2.046

Art 19 2.122, 2.132, 2.134, 2.139, 3.031, 3.034

Art 19(1) $4.347,4.351$

Art 19(2) $2.124,3.031,4.350$

Art 20 3.003, 3.017, 3.026, 3.035,

$4.338,4.339$

Art 20(1) 3.026

Art 20(2) ........ 3.026, 3.030, 3.034, 3.035, $3.160,4.006,4.350$

Art 21 $3.003,3.007,3.017,3.159$, 3.210, 3.213, 3.215, 3.216, 3.229, 4.033, 4.212, 4.221

Art 22 $3.003,4.212,4.221$

Art 23 $3.003,3.235,4.006$ Art 24(1) ......... 4.025, 4.035, 4.036, 4.045 Art 24(2) ......................................... 4.048 Art 25 .............................................. 4.167 Art 25(2) .......................................... 2.095 Art 26(2) 2.092, 4.095

Art 27 4.278

Art 28 4.114

Art 29 4.162

Art 30 $4.279,4.281$

Art 31 4.006, 4.076, 4.212, 4.213-4.220, 4.227

Art 31(1) $4.213,4.214$

Art 31(3) 4.228

Art 32 ... 4.006, 4.076, 4.212, 4.221-4.227 Art 32(1) 4.221, 4.222

Art 32(2) $4.221,4.222$

Art 32(3) 4.221, 4.228

Art 33 4.283, 4.298

Art 34 4.353

Art 35

Art 36 $2.079,3.122$

Art 37 3.017

Art 38 $3.017,3.242$

Art 39 4.284, 4.293

Art 40(2) 4.310 
Art 41(1)

2.024

\section{Rules of the Tribunal}

Art 2 $2.034,2.050,2.076$

Art 5

2.035

Art 7 . 2.041

Art 8(1) 2.062

Art 9 2.041, 4.057

Art 10(1) 2.050

Art 11 2.050

Art 12 2.003

Art 12(1) 2.052

Art 12(2) $2.052,2.126$

Art 13(1) $2.053,2.119$

Art 13(2) 2.053

Art 16(2) $2.036,2.051$

Art 17 2.036

Art 18 $2.040,2.048$

Art 19(3) 4.057

Art 21(1) 2.052

Art 22 2.067

Art 22(4) 2.068

Art 23 2.076

Art 26(3) 2.078

Art 28(6) 2.093

Art 29 2.082

Art 30 4.260

Art 32(1) $2.110,4.004$

Art 33 2.110

Art 35(1) 2.116

Art 35(2) 2.117

Art 36 2.111

Art 36(1)

Art 36(3)

Art 38(4)

Art 40

Art 42(1)

Art 42(2)

Art 42(3)

Art 43

Art 44(3)

Art 45

Art 46

Art 47

Art 49

Art 50

Art 51

Art 52(2) 4.148 4.149 $2.097,4.042$ 4.079 $4.050,4.051$ 5.020 4.056 4.007, 5.020 4.010, 4.011 4.046 $4.047,4.312$
Art 54 4.035

Art 54(1) $4.035,4.036$

Art 54(2) $3.087,4.035,4.036$

Art 54(3) 4.041

Art 54(5) 3.086

Art 55 $4.029,4.031$

Art 56(1) 4.037

Art 56(3) 4.030

Art 59 4.063

Art 59(1) $4.007,4.062,4.278$

Art 59(2) 4.062

Art 59(3) $2.052,4.063$

Art 60 $4.059,4.063$

Art 61 $4.063,4.064$

Art 61(1) 4.059

Art 62 4.061, 4.064

Art 62(1) 4.061

Art 62(4) 4.069

Art 64 4.065

Art 64(2) 2.099

Art 64(3) 2.100

Art 64(4) $2.105,4.282$

Art 65 $4.004,4.066$

Art 66 4.073

Art 67 4.076

Art 67(1) $4.076,4.214,4.215$

Art 67(2) 4.074

Art 67(3) $2.052,4.075$

Art 68

Art 69 $4.077,4.311$

Art 69(1) $4.064,4.085$

Art 71 ... 4.071, 4.072, 4.088, 4.178, 4.245 Art 71(1) 4.070

Art 71(2) $4.070,4.072$

Art 71(5) 4.071

Art 72 2.070, 4.087, 4.119, 4.120, $2.072,4.149$ 
Art 77(1) $4.108,4.115$

Art 77(2) $4.115,4.128$

Art 78(1) 4.120

Art 78(2) 4.127

Art 79 $4.081,4.082,4.122$

Art 80 $4.081,4.122,4.124$

Art 81 $4.115,4.129$

Art 82(1) $4.115,4.128,4.278$

Art 83 4.128

Art 84(1), (3), (4) 4.134

Art 85 4.101

Art 85(2) 2.101

Art 85(3) 2.102

Art 86 $2.103,4.103$

Art 86(1) 4.103

Art 86(4) 4.104

Art 86(5) $4.104,4.125$

Art 87 4.100, 4.101

Art 88 4.107

Art 88(1) 4.108

Art 89 5.011

Art 89(1) 4.169

Art 89(3), (4) 4.171

Art 89(5)

Arts 89-95

4.166, 5.010

Art 90 4.168

Art 90 (1), (2) $4.173,4.174$

Art 90(3) 4.174

Art 90(4) $4.071,4.176,4.178$

Art 91(1), (2) $4.173,4.278$

Art 92 $2.095,4.167$

Art 93 4.279

Art 95 4.179

Art 95(1)

Art 95(2) 4.288

Art 96 4.180

Art 96(1)

Art 96(2)

Art 96(3)

Art 96(4)

Art 96(5), (6)

Art 96(8)

Art 97 3.024, 3.060, 4.004, 4.181, $4.189,4.190,4.192,4.194,4.263$ Art 97(1) ......... 3.083, 4.007, 4.191, 4.194 Art 97(2) 4.194 Art 97(3)
Art 97(4) ............................................ 4.197

Art 97(5) ………................................ 4.198

Art 97(6) .......................................... 4.199

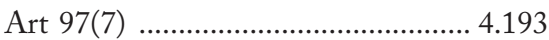

Art 98 ................................... 4.203, 4.208

Art 98(1) ............................. 4.205, 4.208

Art 98(2) .......................................... 4.204

Art 98(3) ........................................... 4.206

Arts 99-104 ...................................... 4.212

Art 99(1) ............................... 4.076, 4.214

Art 99(2) ............................... 4.214, 4.218

Art 99(3) ............................................. 4.216

Art 100 ............................................. 4.222

Art 100(1) ............................. 4.076, 4.222

Art 100(2) ......................................... 4.223

Art 101 ................................ 4.217, 4.226

Art 102 ........... 4.217, 4.218, 4.225, 4.226

Arts 103, 104 ................................... 4.229

Art 105 ........... 4.230, 4.231, 4.278, 5.010

Art 106 ............ 4.230, 4.233, 4.234, 5.010

Art 107 .................................. 4.256, 4.262

Art 108 ................................. 4.256, 4.258

Art 108(3) ......................................... 4.260

Art 108(4) …...................................... 4.261

Art 109 .................................. 4.256, 4.263

Art 109(1), (2) .................................. 4.263

Arts 110-114 .................................. 4.236

Art 110 .............................................. 4.240

Art 110(2) ....... 2.111, 3.137, 4.238, 4.240

Art 110(3) .......................................... 4.238

Art 111 ................................................ 4.093

Art 111(2) ........................................... 4.241

Art 111(4) .............................. 4.004, 4.093

Art 111(6) ....................................... 4.246

Art 112 ...................... 2.094, 4.007, 4.085

Art 112(1) ……………………...... 4.246

Art 112(2) .............................. 2.094, 4.242

Art 112(3) ...... 4.004, 4.084, 4.085, 4.090, 4.246

Art 112(4) .................. 4.004, 4.244, 4.247

Art 113 ...................... 4.004, 4.248, 4.254

Art 113(2) 4.248

Art 113(3) ....... 4.004, 3.138, 4.253, 4.254

Art 114 ..................... 4.004, 4.253, 4.254

Art 117 ...................... 4.265, 4.270, 4.271

Arts 117-121 ........... 4.265, 4.269-4.271, 
Art 118(1)

Art 118(2) $4.271,4.275$

Art 119

Art 120

Art 121

Art 122

Art 123

Art 124

Art 125

Art 126

Art 127

Art 128

Art 128(1)

Art 128(2), (3)

Art 128(4)

Art 128(5)

Art 129(1), (2)

Art 129(3)

Arts 130-137 $4.004,4.302-4.304,4.306$

4.272

$4.270,4.273-4.275$ $4.270,4.276$

$4.265,4.269,4.270,4.277$ $4.265,4.266$

$4.265,4.267,4.268$ $4.279,4.280$ $4.279-4.281,4.353$ 4.299 4.004 4.304 4.305 4.278, 4.303 4.306 4.307 $4.300,4.306$ $3.209,4.310,4.328$
Art 130 $4.310,4.319$

Art 130(1) 4.328

Art 130(2) 3.233

Art 131 $4.313,4.332$

Art 132 4.316

Art 133 $4.320,4.335,4.344$

Art 133(1)

$4.313,4.333$

Art 133(2) $4.321,4.323,4.333$

Art 133(3) $4.322,4.334,4.336$

Art 133(4) 4.320

Art 134 $4.313,4.322,4.334$

Art 135(1), (2) 4.324 Art 135(3) 4.325

Art 137 4.326

Art 138 $3.203,3.209,3.226,3.227$, $3.229,4.327$

Art 138(1) $3.225,3.232$

Art 138(2) $3.230,4.329$

Art 138(3) $3.233,4.328$ 